Office of Naval Research

Department of the Navy

Contract Nonr-220(35)

\title{
NONLINEAR GRAVITY WAVES IN A THIN SHEET OF
} VISCOUS FLUID

by

C. C. Mei
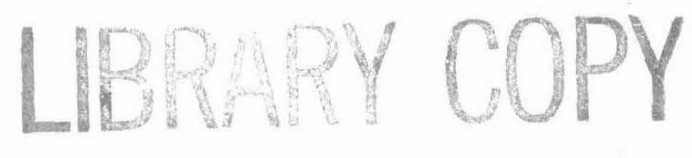

IPF IHE

HYLRODYNAMES LABORATORY

CALIFORNIA INST TUTE OF TECHNOLOGY

PASAOENA 4. CALIFORNIA

Reproduction in whole or in part is permitted for any purpose of the United States Government.

Division of Engineering and Applied Science

California Institute of Technology

Pasadena, California 


\section{NONLINEAR GRAVITY WAVES IN A THIN SHEET OF VISCOUS FLUID}

\section{By C. C. Mei}

1. Introduction. In the study of long gravity waves of finite amplitude, the main body of the existing theories has been built upon the simplifying assumption that the viscosity is either totally negligible, or adequately described by an empirical law. To date very little systematic account of the viscosity effect has appeared that is based on the Navier-Stokes' equations of motion. Thus, in the important problems of flood waves in rivers, Chezy's formula and a variety of empirical laws of hydraulics have been used to replace the viscous stress terms, and this is the approach taken in most of the hydraulic studies on open channel flows. Among theoretical contributions along this line, one may mention the book by Stoker (1957), the works of Dressler (1949), and of Lighthill and Whitham $(1955, \mathrm{I})$. Dressler developed a rigorous theory of roll waves. In particular he obtained a discontinuous periodic solution in the case of relatively large amplitudes and a continuous periodic (cnoidal) solution in the case of small amplitudes. General flood movement in long rivers has been masterfully investigated by Lighthill and Whitham $(1955, \mathrm{I})$, as a type of kinematic waves. Their method of predicting the transient motion of large amplitude waves with discontinuities (or shocks) is especially noteworthy.

Another existing approach to study viscosity in long gravity waves is to assume a shear profile in the basic flow; the wave motion is, however, assumed to be governed by inviscid equations. Although the shear profile can be made as realistic as possible, viscous effects come into the wave theories only implicitly. References may be made to Burns (1953), Hunt (1955), and Benjamin (1962). In particular solitary and periodic cnoidal waves are found by these authors as possible wave forms which move at a steady speed without change of shape.

Rigorous hydrodynamic theory of gravity waves based on Navier-Stokes' equations have been done only in the case of infinitesimal amplitude and relatively deep fluids. A summary of elementary results is given by Wehausen and Laitone (1960). The theories of Yih $(1955,1963)$ and Benjamin (1957) on the instability of a thin sheet of fluid flowing down an inclined plane also belong to the category of infinitesimal waves. $\dagger$

The reasons for lacking a rigorous theory on nonlinear shallow water waves with viscosity is not hard to seek, since the inviscid theory itself has been controversial even after the paper of Ursell (1953) (see Longuet-Higgins (1958)). To state it briefly, there are two basic approaches to the inviscid shallow water theory. In the first, due to Friedrichs (1948), the Airy's theory which predicts the nonexistence of permanent waves is considered to be the first order result of a

$\dagger$ One conclusion from their theories, which is supported by experimental findings of Binnie (1957), is particularly relevant here; that is, the stability of such a flow is governed by disturbances of long wave lengths at low Reynolds numbers up to order unity. 
perturbation theory. The small parameter involved is essentially the ratio between the depth $(H)$ and a horizontal length scale $(L)$ (with $H / L \ll 1$ ). Permanent waves of the solitary and cnoidal types were later found as the second order result in the same perturbation scheme (see Keller (1949)). In the second, Ursell first clarified that, in addition to the above mentioned ratio, the amplitude $a_{0}$ provides a third length scale and, hence, another parameter. He found that Airy's theory belongs properly to the region

$$
H / L \ll 1, \quad\left(a_{0} / L\right)(H / L)^{-3} \gg 1,
$$

the solitary wave and the cnoidal wave to

$$
H / L \ll 1, \quad\left(a_{0} / L\right)(H / L)^{-3}=O(1),
$$

and the linearized tide wave theory to

$$
H / L \ll 1, \quad\left(a_{0} / L\right)(H / L)^{-3} \ll 1 .
$$

Based on Ursell's deductions, Lin and Clark (1959) derived the general equations for long waves over a horizontal bottom in three dimensions. The second approach seems to have received a wider support.

The investigation of the effect of viscosity should naturally be of great importance in shallow liquids, for example, in flood problems or in oceanographic engineering. But the inclusion of viscosity leads to additional complications because of a new length scale, i.e., the viscous diffusion length. In problems of the most practical interest the diffusion length is perhaps very small compared with the fluid depth which is itself small referring to a horizontal length. Thus, the depth is in an awkward position of being large compared to one length but small to another, and the mathematical problem is consequently difficult. A further source of difficulty is that the actual flow is frequently turbulent.

As a first step towards a rigorous understanding of the role of viscosity in long gravity waves, we take the simpler case of high viscosity where the diffusion length is not too small as compared with the fluid depth. In the ordinary theory of flows at a small Reynolds number, it is well known that the pressure gradient and the viscous stress terms control the motion. Here, then, we shall consider the influence of gravity to be of equal importance as those two terms just mentioned. The specific physical problem to be treated concerns with the flow down an inclined plane.

In order to discover non-trivial wave motions in such a fluid, we shall find it necessary to decide first a suitable time scale. This situation is similar to the case of low Reynolds number flow, where one has to use a suitable pressure scale. The formal expansion procedure of Lin and Clark in their development of inviscid long wave theory will be adopted in the present paper. We shall discuss three cases according to the size of the amplitude. If we denote

$$
\epsilon=H / L \text { and } a=\left(\eta^{*}-H\right) / L
$$

where $\eta^{*}$ is the typical surface elevation, then in the first case of $a=O(\epsilon)$ 
we obtain a nonlinear partial differential equation for the dimensionless $\eta$ :

$$
\epsilon^{2} \eta_{t}+\alpha \eta^{2} \eta_{x}=\frac{1}{3} \beta\left(\eta^{3} \eta_{x}\right)_{x} .
$$

The notable feature of this equation is its nonlinear diffusion term on the right. The profile of permanent waves of the type $\eta(x-C t)$ is studied - it is found to be quite similar to the monoclinal flood waves where the Reynolds number is large. The variation of amplitude with wave speed is investigated and the result is supported by a more direct physical argument. It is also found that a critical case exists where the front invades a dry bed.

In the second case we assume $a=O\left(\epsilon^{2}\right)$. The governing equation for $a$ becomes the well-known Burgers' equation which has been thoroughly studied and made use of in gas dynamies. It is worth noting that here the diffusivity is proportional to the square of the Reynolds number and therefore smaller for higher viscosity, whereas in gas dynamics the converse is true (Lighthill (1956)).

In the third case where $a=O\left(\epsilon^{3}\right)$, solitary waves and cnoidal waves occur in the classical theory. We find not only monoclinal waves but waves resembling an undulating hydraulic jump. We also establish a condition whereby undulations may not appear. Periodic waves, and solitary waves are found in the case of vertical wall only.

Comparison with experiments should be desirable but the author is yet unable to find directly usable data from the literature (see Binnie (1957) for references). Furthermore, it is hoped that the effects of surface tension, which would make the mathematies more difficult, will be considered in the future. Some of the results obtained here is, however, independent of this omission.

2. Formulation of the Problem and the Shallow Liquid Expansions. Let us consider the two dimensional laminar flow down a plane inclined at the angle $\theta$ (Fig. 1). We shall assume that the fluid is incompressible and viscous with constant coefficient of viscosity. If the coordinate system is so chosen that the $x$ axis coincides with the inclined bottom and the $y$-axis is normal to it, the continuity and the momentum conservation are expressed by the following three equations:

$$
\begin{aligned}
u_{x}+v_{y} & =0 \\
u_{t}+u u_{x}+v u_{y} & =-\rho^{-1} p_{x}+g \alpha+\nu \nabla^{2} u \\
v_{t}+u v_{x}+v v_{y} & =-\rho^{-1} p_{y}-g \beta+\nu \nabla^{2} v
\end{aligned}
$$

where

$$
\begin{aligned}
& t=\text { time, } u(x, y, t), v(x, y, t)=\text { velocity components } \\
& p(x, y, t)=\text { pressure, } \rho=\text { density, } g=\text { gravitational constant } \\
& \alpha=\sin \theta, \beta=\cos \theta, \nu=\mu / \rho=\text { viscosity coefficient }
\end{aligned}
$$

On the bottom there must be no slip, hence

$$
u=v=0 \quad y=0 .
$$


On the free surface denoted by

$$
y=\eta(x, t),
$$

the kinematic condition requires that

$$
\eta_{t}+u \eta_{x}=v, \quad y=\eta(x, t) .
$$

If we assume the surface to be free of external stress and surface tension, then the components of the stress force in the fluid must vanish on the free surface also (Wehausen and Laitone (1960)), i.e.,

$$
\begin{gathered}
\left(p-2 \mu u_{x}\right) \eta_{x}+\mu\left(u_{y}+v_{x}\right)=0 \\
p-2 \mu v_{y}+\mu\left(u_{y}+v_{x}\right) \eta_{x}=0 .
\end{gathered}
$$

By introducing some characteristic time, length and velocity scales $T, L, U$, respectively, all the variables will now be made dimensionless in the following

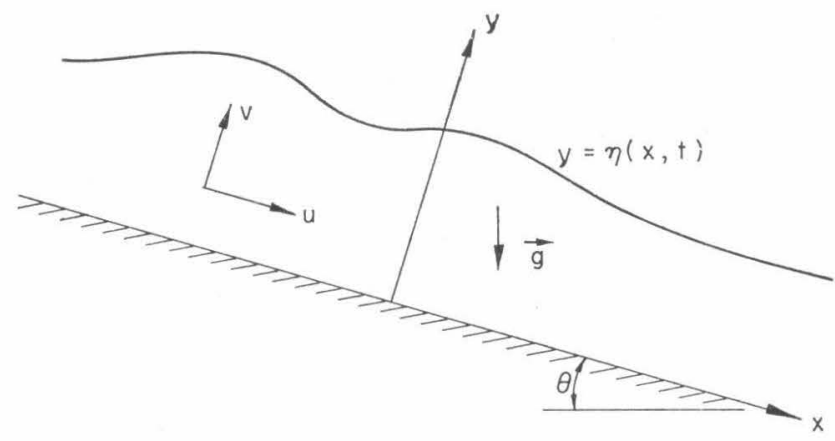

Fig. 1. Nomenclature

manner:

$(u, v) \rightarrow U_{0}(\tilde{u}, v), \quad t \rightarrow T t, \quad(\eta, x, y) \rightarrow L(\eta, x, y), \quad p \rightarrow \mu U_{0} \tilde{p} / L$.

Thus, Eqs. (1), (2), (3), (5), (7), (8) and (9) become

$$
\begin{aligned}
& \tilde{u}_{x}+v_{y}=0 \\
& \tau \tilde{u}_{t}+\tilde{u} \tilde{u}_{x}+v \tilde{u}_{y}=-R^{-1} \tilde{p}_{x}+\alpha F^{-2}+R^{-1} \nabla^{2} \tilde{u} \\
& \tau v_{t}+\tilde{u} v_{x}+v v_{y}=-R^{-1} \tilde{p}_{y}-\beta F^{-2}+R^{-1} \nabla^{2} v \\
& \tilde{u}=v=0 \text { on } y=0 \text {, } \\
& \tau \eta_{t}+\tilde{u} \eta_{x}=v \\
& \left.\left(\tilde{p}-2 \tilde{u}_{x}\right) \eta_{x}+\left(\tilde{u}_{y}+v_{x}\right)=0\right\} y=\eta(x, t) \\
& \left.\left(\tilde{p}-2 v_{y}\right)+\left(\tilde{u}_{y}+v_{x}\right) \eta_{x}=0\right)=\epsilon+a(x, t)
\end{aligned}
$$


In the above equations the customary definitions of Reynolds $(R)$, Froude $(F)$ and Strouhal $(\tau)$ numbers have been used:

$$
R=U_{0} L / \nu, \quad F^{2}=U_{0}^{2} / g L, \quad \tau=L / U_{0} T .
$$

It may be pointed out that the pressure is non-dimensionalized so as to exhibit that the pressure gradient and the viscous stresses are equally dominant when the Reynolds number is small, a fact that is well known in Stokes flow. Here we wish to study the case where the effect of gravity is of comparable importance as those two terms just mentioned. Hence, without loss of generality, we may take

$$
R=F^{2}=\epsilon
$$

This choice determines our length scale $L=\left(U_{\mathrm{G}} \nu / g\right)^{\frac{1}{2}}$. If we further choose

$$
U_{0}=(g H)^{\frac{1}{2}}
$$

where $H$ is a typical depth of the fluid, we have

$$
L=(H / g)^{\frac{1}{3}} \nu^{\frac{1}{2}} \text { and } \epsilon=H / L=\left(H^{3} g\right)^{\frac{1}{3}} \nu^{-\frac{1}{2}} .
$$

There exists an elementary solution to the governing equations which will be called the primary flow where

$$
\partial / \partial t=\partial / \partial x=v=0, \quad \eta=\epsilon, \quad \tilde{u}=U(y), \quad \tilde{p}=P(y) .
$$

From Eqs. (12) and (13) we have, respectively,

$$
U_{y y}+\alpha=0, \quad P_{y}+\beta=0,
$$

with

$$
U_{y}=0, \quad P=0, \quad y=\epsilon \text { and } \quad U=0, \quad y=0 .
$$

The solution is easily found to be

$$
U(y)=\alpha \epsilon^{2}\left(y / \epsilon-y^{2} / 2 \epsilon^{2}\right)
$$

and

$$
P(y)=\beta \epsilon(1-y / \epsilon) .
$$

Equation (22) indicates that the typical magnitude of the speed involved here is really of the order $\epsilon^{2}(g H)^{\frac{1}{2}}$ and not $\sqrt{g H}$ as used in Eq. (20). But the present choice is made so that the parameter $\epsilon=H / L$ is conveniently introduced as the Froude number (cf. (19)). A natural time scale is such that

$$
L / T=\epsilon^{2}(g H)^{\frac{1}{2}}
$$

which, by Eq. (21), implies that

$$
T=\nu^{\frac{2}{3}} H^{-\frac{7}{4}} g^{-\frac{5}{4}} .
$$

Going back to Eq. (18), we find that the Strouhal number should then be

$$
\tau=\epsilon^{2} .
$$


This choice is by no means trivial, and a way of obtaining it is given in Appendix I.

Putting

$$
\tilde{p}=P+p \text { and } \tilde{u}=U+u
$$

we have from $(11-17)$

$$
\left.\begin{array}{rl}
u_{x}+v_{y}=0 & \\
\epsilon^{3} u_{t}+\epsilon\left\{(U+u) u_{x}+\left(U_{y}+u_{y}\right) v\right\} & =-p_{x}+\nabla^{2} u \\
\left.\epsilon^{3} v_{t}+\epsilon_{\{}(U+u) v_{x}+v v_{y}\right\} & =-p_{y}+\nabla^{2} v \\
u=v=0, \quad y & =0, \\
\epsilon^{2} \eta_{t}+(U+u) \eta_{x} & =v \\
\left(P+p-2 u_{x}\right) \eta_{x}+U_{y}+u_{y}+v_{x} & =0 \\
P+p-2 v_{y}+\left(U_{y}+u_{y}+v_{x}\right) \eta_{x} & =0
\end{array}\right\} y=\eta(x, t) .
$$

We now state explicitly the fundamental assumption that the depth of fluid is small, i.e.,

$$
\epsilon=H / L \ll 1
$$

and the amplitude of free surface is at most as large as $O(\epsilon)$. This provides a limit of applicability of the present studies. (For example, in heavy oils $\nu \cong$ $10^{-2} \mathrm{ft}^{2} / \mathrm{sec}$. If we let $\epsilon=0.1$, the depth $H$ is of order $10^{-3} \mathrm{ft}$ and $U_{0} \epsilon^{2} \cong 1$ in./min.). It also enables us to introduce the following expansions in powers of $y$.

$$
\begin{aligned}
& \psi(x, y, t)=\sum_{0} y^{n} \psi^{(n)}(x, t) \\
& p(x, y, t)=\sum_{0} y^{n} p^{(n)}(x, t),
\end{aligned}
$$

where $\psi$ denotes the stream function such that

$$
u=\psi_{y}, \quad v=-\psi_{x} .
$$

The first two terms in the series (36) can be quickly disposed,

$$
\psi^{(0)}=\psi^{(1)}=0
$$

because of (31). Recursive relations will be found by substituting the series in Eqs. (26) and (27) and comparing the like powers of $y$ (see Appendix II) so that $\psi^{(n)}$ and $p^{(n)}$ can be related to $\psi^{(2)}$ and $p^{(0)}$. The results are as follows:

$$
\begin{gathered}
p_{x}^{(0)}=6 \psi^{(3)}, \quad p^{(1)}=-2 \psi_{x}^{(2)}, \quad p^{(2)}=-\frac{1}{2} p_{x x}^{(0)} \\
12 \psi^{(4)}=-2 \psi_{x x}^{(2)}+\epsilon^{2} \psi_{t}^{(2)}, \text { etc. }
\end{gathered}
$$

These recursion relations can then be used to derive from the three boundary conditions (32)-(34), three partial differential equations for three unknowns $\psi^{(2)}, p^{(0)}$ and $\eta$ (or $a$ with $\eta=\epsilon+a$ ). For practical reasons, only terms of the first few orders will be kept in these equations, and this is systematically done 
according to the size of the amplitude $a$ for the following three cases: $a=O(\epsilon)$, $O\left(\epsilon^{2}\right)$ and $O\left(\epsilon^{3}\right)$.

Equations $(40)-(43)$ also indicate that $p^{(0)}, p^{(1)} \cdots \psi^{(2)}, \psi^{(3)} \cdots$ etc. are all of comparable size; the precise order of magnitude can be expressed in terms of the free surface amplitude. Substituting the series (36) and (37) into (33) and making use of (22) and (23), one finds that the leading terms are

$$
U_{y}+u_{y}+\cdots=-\alpha a+2 \psi^{(2)}+\cdots=0, \quad y=\eta .
$$

Hence, $\psi^{(2)}=O(a)$. Similarly from the leading terms of (34)

$$
P+p+\cdots=-\beta a+p^{(0)}+\cdots=0, \quad y=\eta,
$$

one gets $p^{(0)}=O(a)$. We may thus conclude that

$$
p^{(0)}, p^{(1)}, p^{(2)}, \cdots \psi^{(2)}, \psi^{(3)}, \psi^{(4)}, \cdots=O(a) .
$$

3. Large Amplitude Waves $a=O(\eta)=O(\varepsilon)$. In this case it is convenient to use $\eta$ throughout instead of $\alpha$. To demonstrate the procedure of argument that is to be used repeatedly in this paper, the order of the leading terms will be written beneath them. Thus, from the first boundary condition (32), we have

$$
\begin{array}{r}
\epsilon^{2} \eta_{t}+\eta_{x}\left[\alpha \epsilon^{2}\left(\frac{\eta}{\epsilon}-\frac{\eta^{2}}{2 \epsilon^{2}}\right)+2 \eta \psi^{(2)}+3 \eta^{2} \psi^{(3)}+\cdots\right] \\
O\left(\epsilon^{3}\right) O(\epsilon)\left[\quad O\left(\epsilon^{2}\right) \quad O\left(\epsilon^{2}\right) \quad O\left(\epsilon^{3}\right) \quad\right] \\
+\eta^{2} \psi_{x}^{(2)}+\eta^{3} \psi_{x}^{(3)}+\cdots=0 \\
O\left(\epsilon^{3}\right) \quad O\left(\epsilon^{4}\right)
\end{array}
$$

If we decide to be accurate up to the order $O\left(\epsilon^{4}\right)$, all the terms appearing above must be retained. Furthermore, the unknowns $\psi^{(2)}$ and $\psi^{(3)}$ can be expressed in terms of $\eta$ by making use of the other two boundary conditions. To do so in accordance with the desired accuracy, we must obtain $\psi^{(2)}$ valid up to the order $O\left(\epsilon^{2}\right)$ and $\psi^{(3)}$ up to $O(\epsilon)$. From (33) and (34) it follows that

$$
\begin{aligned}
\left\{-\beta a+p^{(0)}+\eta p^{(1)}+\cdots-\right. & \left.2\left[2 \eta \psi_{x}^{(2)}+\cdots\right]\right\} \eta_{x} \\
& \underline{-\alpha a}+\underline{2 \psi^{(2)}}+\underline{6 \eta \psi^{(3)}}+\cdots-\eta^{2} \psi_{x x}^{(2)}-\cdots=0,
\end{aligned}
$$

and

$\underline{-\beta a}+\underline{p^{(0)}}+\eta p^{(1)}+\cdots+2\left(2 \eta \psi_{x}^{(2)}+\cdots\right)$

$$
+\left\{-\alpha a+2 \psi^{(2)}+\cdots-\eta^{2} \psi_{x x}^{(2)}-\cdots\right\} \eta_{x}=0 .
$$

Collecting only the leading terms (underlined) in the above two equations, we get

$$
p^{(0)}=\beta a+O\left(\epsilon^{2}\right), \quad 2 \psi^{(2)}=\alpha a-6 \eta \psi^{(3)}+O\left(\epsilon^{3}\right)
$$

Because of (40) we have

$$
\psi^{(3)}=\frac{1}{6} p_{x}^{(0)}=\frac{1}{6} \beta a_{x}+O\left(\epsilon^{2}\right)=\frac{1}{6} \beta \eta_{x}+O\left(\epsilon^{2}\right) .
$$


Hence,

$$
2 \psi^{(2)}=\alpha(\eta-\epsilon)-\beta \eta \eta_{x}+O\left(\epsilon^{3}\right),
$$

and (45) becomes, upon substitution,

$$
\epsilon^{2} \eta_{t}+\alpha \eta^{2} \eta_{x}=\frac{1}{3} \beta\left(\eta^{3} \eta_{x}\right)_{x}+O\left(\epsilon^{5}\right) .
$$

Equation (46) is a nonlinear partial differential equation not yet seen in the literature. To discuss it, we first ignore the right hand side which is of $O\left(\epsilon^{4}\right)$; thus,

$$
\epsilon^{2} \eta_{t}+\alpha \eta^{2} \eta_{x}=O\left(\epsilon^{4}\right) \text {. }
$$

This is an equation belonging to a general class of "kinematic waves"

$$
\eta_{t}+V(\eta) \eta_{x}=\eta_{t}+q_{x}=0
$$

where for the present case

$$
V=\alpha(\eta / \epsilon)^{2} \text { and } q=\frac{1}{3} \eta^{3} / \epsilon^{2}
$$

Many nonlinear physical phenomena are governed by "conservation laws" of this type to the crudest approximation which have been thoroughly studied by Lighthill and Whitham (1955, I and II) with regard to flood waves and traffic flows. Several salient features of Eq. (47) may be recalled. It possesses straight line characteristics in the $x-t$ plane:

$$
d x / d t=V(\eta)
$$

along which $\eta=$ constant. Hence, for any given initial data $\eta(x, 0)=\eta_{0}(x)$ the solution is uniquely given by

$$
x=\xi+V\left(\eta_{0}(\xi)\right) t
$$

until the crossing of characteristics occurs. Due to the nonlinearity, crossing will inevitably occur for some initial disturbance, leading physically to the breaking of waves. If discontinuous solutions, i.e., shocks or bores, are admitted, one can, with the aid of a suitable shock condition, solve the initial value problem completely. The discontinuity is, of course, the idealization of a narrow zone of drastic changes. Within this zone some terms of higher order may no longer be ignored, and a better approximation must be sought to study the shock structure which can then be fitted into the discontinuity. For this reason, and for the analysis of weak shocks which any strong shock eventually reduces to, one must go back to Eq. (46).

3a. Permanent Waves. The simplest non-trivial solution of Eq. (46) is of the type

$$
\eta(x, t)=\eta(x-C t)=\eta(\xi)
$$

so that the wave profile moves at the constant speed $C$ without change of form. Upon changing

$$
\partial / \partial t \rightarrow-C \partial / \partial \xi, \quad \partial / \partial x \rightarrow \partial / \partial \xi
$$


and integrating once with respect to $\xi$, Eq. (46) is simplified to an ordinary differential equation

$$
\frac{1}{3} \beta \eta^{3} \eta_{\xi}=\frac{1}{3} \alpha \eta^{3}-C \epsilon^{2} \eta+D .
$$

The constant of integration can be chosen by requiring that $\eta_{\xi}=0$ when $\eta=\epsilon$. Hence,

$$
\frac{1}{3} \beta \eta^{3} \eta_{\xi}=\frac{1}{3} \alpha\left(\eta^{3}-\epsilon^{3}\right)-C \epsilon^{2}(\eta-\epsilon),
$$

or

$$
\begin{aligned}
\eta_{\xi} & =\left(\alpha / \beta \eta^{3}\right)(\eta-\epsilon)\left[\eta^{2}+\epsilon \eta+\epsilon^{2}(1-3 C / \alpha)\right] \\
& =-\left(\alpha / \beta \eta^{3}\right)\left[(\eta-\epsilon)\left(H_{1}-\eta\right)\right]\left(\eta+H_{2}\right)
\end{aligned}
$$

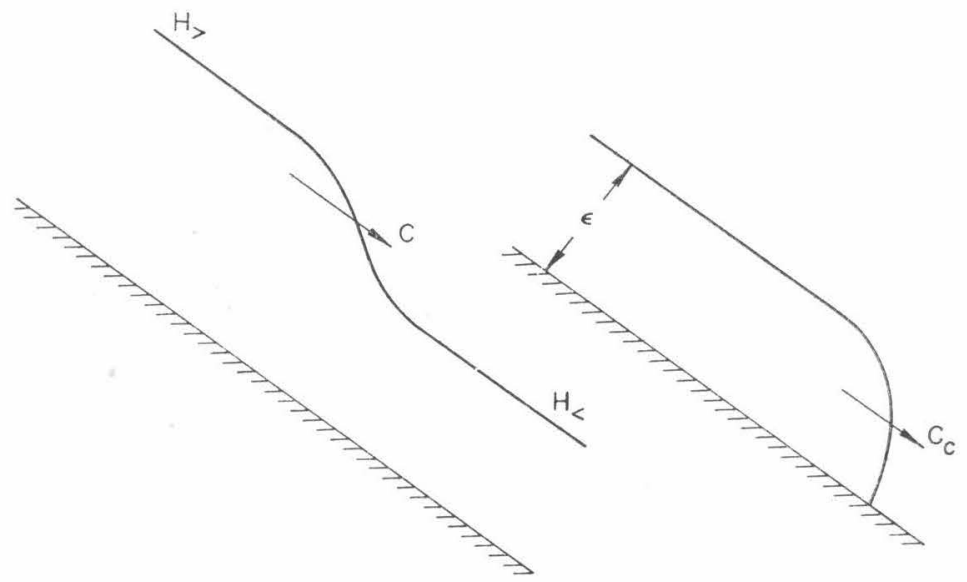

FIG. 2. Monoclinal wave of large amplitude $(a=O(\epsilon))$. a). Normal case where $H_{\gtrless} \underset{\min }{\max \left(\epsilon, H_{1}\right)}$ b). Critical case.

where

$$
\begin{aligned}
& H_{1}=\frac{1}{2} \epsilon[-1+\sqrt{3(4 C / \alpha-1)}], \\
& H_{2}=\frac{1}{2} \epsilon[1+\sqrt{3(4 C / \alpha-1)}]
\end{aligned}
$$

Clearly, the value of $\eta$ lies between $\epsilon$ and $H_{1}$, i.e.,

and from (50),

$$
\begin{aligned}
& \text { if } H_{1}>\epsilon \text { then } H_{1}>\eta>\epsilon \\
& \text { if } H_{1}<\epsilon \text { then } H_{1}<\eta<\epsilon
\end{aligned}
$$

$$
\eta_{\xi}<0
$$

implying that the surface declines monotonically with $\xi$. The wave profiles corresponding to (53.a) and (53.b) are obtained and shown in Fig. (2a). Since the profile connects two levels, the larger of the two $\left(H_{1}, \epsilon\right)$ being the upstream level and the smaller being the downstream level, it can be used as the shock 
structure for a given shock strength. It also stands on its own as a possible steady wave profile which can properly be called the "monoclinal wave"; the name is suggested by a somewhat similar situation in river waves (Lighthill and Whitham, 1955, I). It is well known that when the frictional effect on the river bottom is approximated by Chezy's formula, a steady wave profile may exist in the

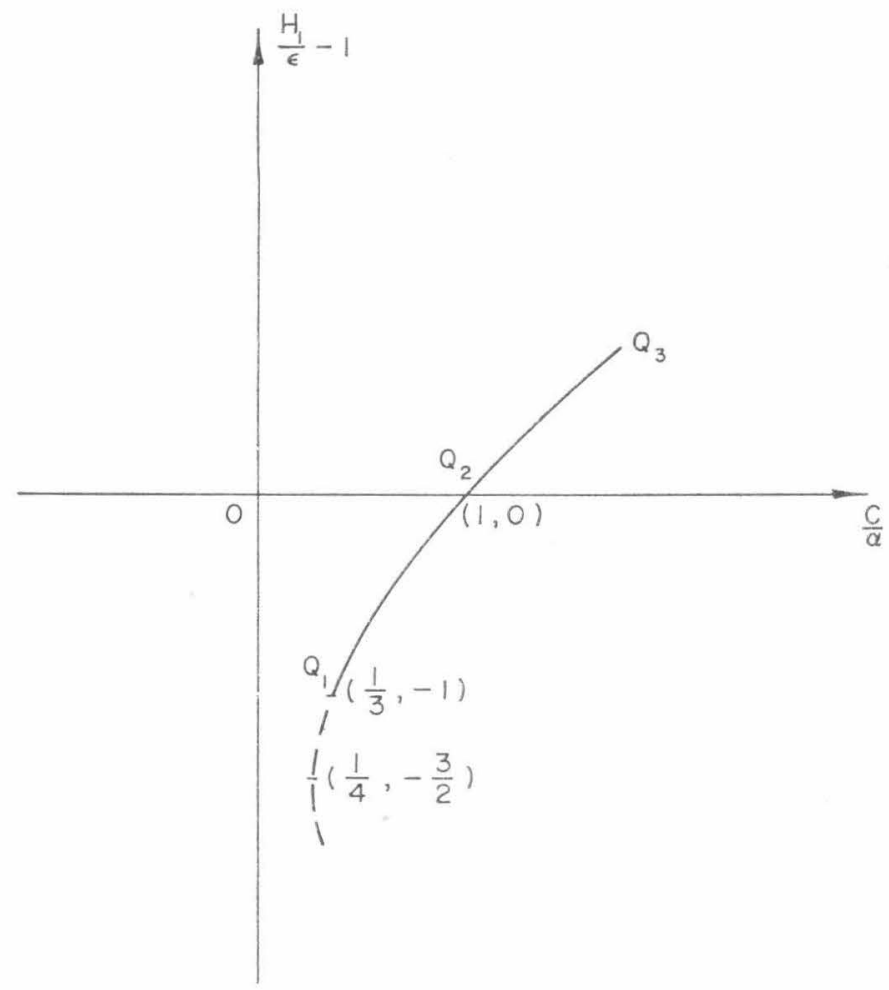

Frg. 3. Amplitude-Wavespeed relation

water flowing down a sloping channel where the governing differential equation is

$$
\eta_{\xi}=-S \frac{\left(\eta-h_{0}\right)\left(h_{1}-\eta\right)(\eta-H)}{\eta^{3}-h_{c}^{3}} h_{i}>\eta>h_{0}>H .
$$

When $\eta>h_{c}$ Eq. (55) behaves remarkably like Eq. (50).

Equation (51) is an explicit relation between the wave speed $C$ and the height $H_{1}$. Since $\left|H_{1}-\epsilon\right|$ is the maximum amplitude, one may follow the terminology of Lighthill and Whitham and call this the "amplitude dispersion relation" $\dagger$ in contrast with the ordinary frequency dispersion relation of linear waves. The plot of $H_{1} / \epsilon$ and $C / \alpha$ is shown in Fig. (3) which is a parabola. Since $H_{1} / \epsilon$ has to be positive, only the branch $Q_{1} Q_{2} Q_{3}$ has physical significance. It is seen that the smallest wave speed $C / \alpha=1 / 3$ is associated with $H_{1}=0$ which corresponds to a

$\dagger$ It corresponds to the Rankine-Hugoniot relations of gas dynamics. 
bore invading a dry bed (Fig. 2b). At the front of the bore $(\eta=0)$ the free surface (according to Eq. (50)) is normal to the bed $\left(\eta_{\xi}=\infty\right)$. A still better approximation is therefore needed if one wants a truer description of the neighborhood of such a bore front. Nevertheless, the velocity $C_{c}=\alpha / 3$ is the precise steady velocity at which a thin sheet of oil may advance down a dry bed as will be shown shortly by the elementary argument of mass conservation. In dimensional form, we have

$$
C_{c}^{*}=L C_{c} / T=\frac{1}{3} \alpha \epsilon^{2} \sqrt{g H}=\frac{1}{3} \alpha g H^{2} / \nu .
$$

It is also seen from Fig. (3) that $C / \alpha$ increases monotonically with $H_{1} / \epsilon$ (keeping in mind that the larger of the two $\left(H_{1}, \epsilon\right)$ refers to the upstream and the smaller to the downstream). When $H_{1} \cong \epsilon$ the amplitude of the monoclinal wave is infinitesimal and we obtain the result that

$$
C=C_{i}=\alpha \quad \text { or } C_{i}^{*}=\alpha g H^{2} / \nu .
$$

At the same point the slope of the amplitude dispersion curve is unity as is easily obtained from (51). Hence, the quantity $C_{i}^{*}$ plays the same role as $\sqrt{g H}$ in the classical long wave theory for an inviscid fluid. $\ddagger$

The explicit solution of (50) is easily found to be

$$
e^{\alpha \xi / \beta}=e^{\eta / \epsilon}\left(H_{1}-\eta\right)^{S_{1}}(\eta-\epsilon)^{-S_{2}}\left(\eta+H_{2}\right)^{S_{3}}
$$

where

$$
S_{1}=H_{1}^{3}\left[\epsilon\left(H_{1}-\epsilon\right)\left(\epsilon+H_{2}\right)\right]^{-1}, \quad S_{2}=\epsilon^{2}\left[\left(H_{1}-\epsilon\right)\left(\epsilon+H_{2}\right)\right]^{-1} \quad(58 . \mathrm{b}, \mathrm{c})
$$
and

$$
S_{3}=H_{2}^{3}\left[\epsilon\left(\epsilon+H_{2}\right)\left(H_{1}+H_{2}\right)\right]^{-1} .
$$

Equation (51) as obtained here after numerous approximations is also the exact consequence of mass conservation. Consider the case where the surface elevation changes from $\epsilon(1+Z)$ far upstream to $\epsilon$ far downstream (the conjugate case of $\epsilon$ to $\epsilon(1-|Z|)$ is similar). In the frame of reference where the wave front is stationary, the velocity profiles at far upstream and far downstream are (cf. Eq. (22))

$$
\begin{gathered}
U_{-}-C \epsilon^{2}=\alpha \epsilon^{2}(1+Z)^{2}\left\{y / \epsilon(1+Z)-y^{2} / 2 \epsilon^{2}(1+Z)^{2}\right\}-C \epsilon^{2} \\
U_{+}-C \epsilon^{2}=\alpha \epsilon^{2}\left\{y / \epsilon-y^{2} / 2 \epsilon^{2}\right\}-C \epsilon^{2}
\end{gathered}
$$

In the above we have taken into account that $C$ and $U$ are nondimensionalized with different reference scales, i.e., $L / T$ and $\sqrt{g H}$ respectively, and that $L / T=$ $\epsilon^{2} \sqrt{g H}$ from Eq. (24). Since the mass flux must be constant, we can equate the corresponding discharges,

$$
Q_{-}=\int_{0}^{\epsilon(1+z)}\left(U_{-}-C \epsilon^{2}\right) d y=Q_{+}=\int_{0}^{\epsilon}\left(U_{+}-C \epsilon^{2}\right) d y .
$$

$\ddagger$ This result checks with the infinitesimal wave theory, see Benjamin (1957, Eq. (2.4)) 
Upon substituting (59) into the preceding equation and carrying out the integration, we get

$$
C=\frac{1}{2} \alpha\left[1+(1+Z)+(1+Z)^{2}\right] .
$$

It then follows that,

$$
1+Z=\frac{1}{2}[-1+\sqrt{3(4 C / \alpha-1)}] .
$$

In the case where the surface elevation at $\xi \rightarrow-\infty$ is $\epsilon$, one simply interprets Eqs. (61) and (62) by allowing $Z$ to have negative values. Then $|Z|$ represents the difference in descent of surface from $\epsilon$ to $\epsilon(1-|Z|)$. Hence, $(1+Z)$ can be identified with $H_{1} / \epsilon$ in Eq. (51). This simple derivation, of course, furnishes no information on the details of the transition region. Since Eq. (62) concerns only with surface heights at infinities where $\partial / \partial \xi=\partial^{2} / \partial \xi^{2}=0$, it is not affected by the presence of surface tension had the latter been assumed to be significant. Furthermore, Eq. (62) holds as long as the flow is laminar, i.e., it does not subject to the limitations of any approximation.

3b. Similarity of the General Equation. It would certainly be desirable to solve the full partial differential equation (46) exactly, since its solution would be valuable especially in studying the decay of an initially strong disturbance. The possibility of having similarity solutions is pointed out here to prepare for future research. As can be shown readily, Eq. (46) is invariant under the transformation

$$
\eta \rightarrow \gamma \eta, \quad x \rightarrow \gamma x, \quad t \rightarrow t / \gamma .
$$

Hence, we can take

$$
\eta(x, t)=x f(\zeta)=F(\zeta) / t
$$

where

$$
\zeta=x t
$$

Differentiating, we have

$$
\eta_{t}=x^{2} f^{\prime}, \quad \eta_{x}=f+\zeta f^{\prime}, \quad \eta_{x x}=2 t f^{\prime}+\zeta t f^{\prime \prime}
$$

It follows that upon substitution Eq. (46) may be written as

$$
\epsilon^{2} f^{\prime}+\alpha f^{2}\left(f+\zeta f^{\prime}\right)=\beta\left[f^{2}\left(f+\zeta f^{\prime}\right)^{2}+\frac{1}{3} \zeta f^{3}\left(2 f^{\prime}+\zeta f^{\prime \prime}\right)\right],
$$

which is a nonlinear ordinary differential equation. For any boundary value problem in which the boundary conditions are also similar, i.e., they are invariant under the same transformation (63), exact integration can be done at least numerically.

3c. Further Remarks About the "Conservation Law" (47). General nonsteady solution of Eq. (46) is not easy to find: but, as has been pointed out earlier, the

$\dagger$ Along any hyperbolic path $x t=\zeta=$ constant, the slope $\eta_{x}$ remains the same. 
more approximate equation, obtained by omitting the right hand side of Eq. (46), lends itself to analytical investigations. We shall mention several general facts about its discontinuous solutions which are of relevance here.

For any conservation law a shock condition can be derived

$$
[q]=C[\eta]
$$

connecting the jumps ([ ] in $\eta$ and $q$, and the shock speed $C$. Hence, in the present case,

$$
C=\frac{1}{3} \frac{\alpha}{\epsilon^{2}} \frac{\eta_{-}^{3}-\eta_{+}^{3}}{\eta_{-}-\eta_{+}}=\frac{1}{3} \frac{\alpha}{\epsilon^{2}}\left(\eta_{-}^{2}+\eta_{-} \eta_{+}+\eta_{+}^{2}\right)
$$

where the subscripts "-_" and "+" refer respectively to the upstream and downstream side of the shock. This relation is completely equivalent to Eq. (61), suggesting that (47) and (68) represent the simple fact of mass conservation. It is well known that a question of uniqueness is associated with the conservation laws (see (e.g.) Lax (1957), Courant and Hilbert (1963), or Jeffrey and Taniuti (1964)), i.e., for the same prescribed initial data there exist many discontinuous (weak) solutions. $\uparrow$ The correct choice can usually be decided by physical considerations. For example, in inviscid gas dynamies involving shocks this is achieved by requiring the entropy to increase from the upstream to the downstream side of the shock, whereas in water wave theory involving bores or hydraulic jumps the energy should decrease across the shock. Alternatively, one can obtain the correct weak solution by retaining viscosity terms (generally of higher order derivatives) and finally taking the limit of small viscosity (Lighthill (1956)). In the present example the corresponding "viscosity" term is the one on the right hand side of Eq. (46), and the monoclinal solution is obtained without neglecting it. Since the profile of the monoclinal wave, representing also the shock structure, always changes from a higher elevation to a lower one, we conclude that across the discontinuity in the weak solution of Eq. (47) we must have

$$
\eta_{-}>\eta_{+}
$$

This provides a further condition to render the problem determinate. The same conclusion can also be obtained by imposing a mathematical condition that a discontinuity is permissible if it indeed represents the breaking of a continuous solution (Courant and Hilbert (1962) p. 151), i.e., each point of the shock curve in the $x-t$ plane is crossed by two forward-drawn characteristics, one from each side of the shock. This implies analytically that

$$
V_{-}>C>V_{+} \text {. }
$$

It is easy to see that the shock speed as deduced from the jump condition Eq. (69) satisfies this condition if (70) holds.

$\dagger$ Precisely the same equation (47) is used in Jeffrey and Taniuti to demonstrate this point (p. 119). 
4. Medium Amplitude Waves, $a=O\left(\varepsilon^{2}\right)$. In this case the leading terms, e.g., $\left(\epsilon^{2} \eta_{t}\right)$, in Eq. (45) are of the order $O\left(\epsilon^{4}\right)$. If we want the accuracy up to $O\left(\epsilon^{5}\right)$ only, then the terms kept in the analysis of $\$ 3$ are more than adequate. Thus, we may take the result of Eq. (46) and simplify it by noting that

$$
\eta=\epsilon+a, \quad \eta^{2}=\epsilon^{2}+2 \epsilon a+O\left(\epsilon^{4}\right), \quad \eta^{3}=\epsilon^{3}+O\left(\epsilon^{4}\right),
$$

and obtain instead

$$
\epsilon^{2} a_{t}+\alpha\left(\epsilon^{2}+2 \epsilon a\right) a_{x}=\frac{1}{3} \beta \epsilon^{3} a_{x x}+O\left(\epsilon^{6}\right) .
$$

Letting

$$
A=a+\epsilon / 2 \text { and } \tilde{t}=2 \alpha t / \epsilon
$$

we obtain

$$
A_{\bar{t}}+A A_{x} \cong\left(\beta \epsilon^{2} / 6 \alpha\right) A_{x x}
$$

which is the well-known Burgers' equation. Its equally well-known solution for the initial value problem (Hopf (1950) Cole (1951)) has been used extensively by Lighthill (1956) to study viscosity effects in sound waves of finite amplitude. In the absence of such a general solution of Eq. (46), the Hopf-Cole solution is useful to analyze the decay of large amplitude waves when the assumption $a=O\left(\epsilon^{2}\right)$ is satisfied, which is the eventual situation of most waves.

The permanent wave solution for (72), corresponding to $a=0$, at $a_{\xi}=0$, is well known to be of the monoclinal type

$$
a / \epsilon=Z /[1+\exp [(3 \alpha / \beta \epsilon) Z \xi], \quad \xi=x-C t,
$$

where

$$
Z=C / \alpha-1
$$

Hence, if $C / \alpha-1 \gtrless 0$, the surface profile lowers from $\left(\underset{\epsilon}{\epsilon^{(1+Z)}}\right)$ at upstream to $(\epsilon \in(1-|z|)$ at downstream (Fig. (4)). The amplitude variation with wave speed is now given by Eq. (76) which represents a straight line. The slope of the "amplitude dispersion curve" ( $Z$ vs. $C / \alpha)$ is unity. Because of the prerequisite $a=O\left(\epsilon^{2}\right)$, only the portion of the dispersion curve lying within a narrow belt is of acceptable validity. Thus, while the dispersion curve of $\$ 3$.a is the exact one, Eq. (76) is but an approximation near $Z=0$ by the straight tangent there.

The width of the region of relatively rapid changes is measured by

$$
(\beta \epsilon / 3 \alpha)(1 /|Z|) \text {. }
$$

Thus, the wave front is steeper for a large difference in elevations, or a higher viscosity (small $\epsilon)$, or a larger inclination $(\theta \rightarrow \pi / 2, \beta / \alpha \rightarrow 0)$.

An important transient property of Burgers' equation may be mentioned (Cole, 1951), that the diffusion term on the right hand side smoothes out even the strongest initial discontinuity although the nonlinear convection term tends to steepen it. In particular, an initial step in $A, A(x, 0)=+A_{0} H(-x)$, is 
gradually smoothed out to the steady state solution of (75) like

$$
\exp \left(-A_{0}^{2} \tilde{t} / 16 \kappa\right) \text {. }
$$

where $\kappa$ is the "diffusivity"

$$
\kappa=\beta \epsilon^{2} / 3 \alpha .
$$

In the present case, if the initial surface profile is a step function of the height $A_{0}=\epsilon Z$, it will smooth out to the shape described by (75) with the deviation dying out with time like

$$
e^{-t / T_{0}}
$$

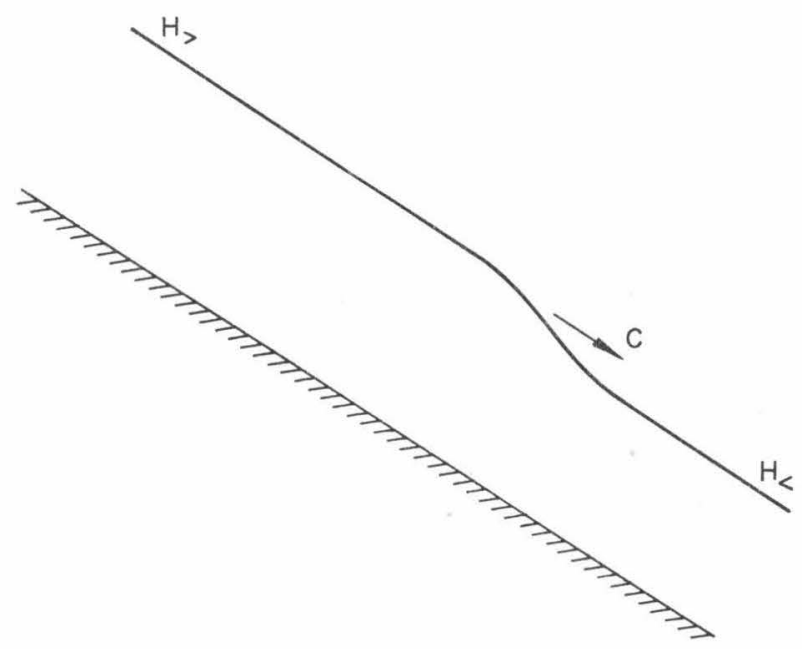

FIG. 4. Monoclinal wave of medium amplitude $\left(a=O\left(\epsilon^{2}\right)\right)$ where $H \gtrless-\frac{\max (\epsilon, \epsilon(1+Z))}{\min }$.

where

$$
T_{0}=8 \beta \epsilon^{3} / 3 \alpha^{2} A_{0}^{2},
$$

can be called the relaxation time. Thus, the decay is faster for stronger initial discontinuity (larger $A_{0}$ ) and higher viscosity (or smaller depth, smaller $\epsilon$ ). The decay rate also depends on the angle of inclination

$$
T \alpha \beta / \alpha^{2}=\cos \theta / \sin ^{2} \theta
$$

thus,

$$
T_{0} \rightarrow \infty, \quad \theta \rightarrow 0 \quad \text { and } \quad T_{0} \rightarrow 0, \quad \theta \rightarrow \pi / 2 .
$$

5. Small Amplitude Waves $a=O\left(\varepsilon^{3}\right)$. I is well known in the theory of long gravity waves in a perfect fluid that in the range of $a=O\left(\epsilon^{3}\right)$, solitary waves (non-periodic) and cnoidal waves (periodic) exist; and, in developing a theory 
for them, better approximations must be made than in the large amplitude case. To search for possible waves in the present case of high viscosity, we write down the kinematic boundary condition again,

$$
\begin{aligned}
\frac{\epsilon^{2} a_{t}}{O\left(\epsilon^{5}\right)}-\frac{a_{x}\left\{\frac{\alpha \epsilon^{2}}{2}\left[1-\left(\frac{a}{\epsilon}\right)^{2}\right]\right.}{O()^{3}\left\{O\left(\epsilon^{2}\right) O\left(\epsilon^{6}\right) \quad\right.}+\frac{2 \eta \psi^{(2)}}{O(\epsilon)^{4}} & \left.=3 \eta^{2} \psi^{(3)}+\cdots\right\} \\
& +\frac{\eta^{2} \psi_{x}^{(2)}}{O\left(\epsilon^{5}\right.}+\frac{\eta^{3} \psi_{x}^{(3)}}{O\left(\epsilon^{6}\right)}+\frac{\eta^{4} \psi_{x}^{(4)}}{O\left(\epsilon^{7}\right)}+\cdots=0
\end{aligned}
$$

in which $\psi^{(3)}$ and $\psi^{(4)}$ can be eliminated through (40) and (43). Only terms of order up to $O\left(\epsilon^{7}\right)$ will be kept. We will also attempt to transform (83) to a single equation for $a$; hence, we must express $\psi^{(2)}, \psi^{(3)}$, and $\psi^{(4)}$ in terms of a valid up to the order $O\left(\epsilon^{5}\right), O\left(\epsilon^{4}\right)$ and $O\left(\epsilon^{3}\right)$, respectively. From the boundary conditions (33) and (34), it follows that

$$
\begin{aligned}
& a_{x}\left\{-\beta a+p^{(0)}+\cdots-2\left[2 \eta \psi_{x}^{(2)}+\cdots\right]\right\} \\
& O\left(\epsilon^{3}\right)\left\{O\left(\epsilon^{3}\right) \quad O\left(\epsilon^{4}\right) \quad\right\} \\
& \left.+\frac{[-\alpha a}{O\left(\epsilon^{3}\right)}+\frac{2 \psi^{(2)}}{O\left(\epsilon^{3}\right)}+\frac{6 \eta \psi^{(3)}}{O\left(\epsilon^{4}\right)}+\frac{12 \eta^{2} \psi^{(4)}}{O\left(\epsilon^{5}\right)}+\cdots-\frac{\eta^{2} \psi_{x x}^{(2)}}{O\left(\epsilon^{5}\right)}-\cdots\right]=0
\end{aligned}
$$

and

$$
\begin{aligned}
\frac{[-\beta a}{O\left(\epsilon^{3}\right)}+\frac{p^{(0)}}{O\left(\epsilon^{3}\right)}+\frac{\eta p^{(1)}}{O\left(\epsilon^{4}\right)}+\cdots+\frac{2\left(2 \eta \psi_{x}^{(2)}\right.}{O\left(\epsilon^{4}\right)} & +\cdots)] \\
& +a_{x}\left(-\alpha a+2 \psi^{(2)}+\cdots\right)=0 \\
& O\left(\epsilon^{3}\right)\left(O\left(\epsilon^{3}\right)+\cdots\right)
\end{aligned}
$$

Collecting the underlined terms which are of major importance, we have from (84)

$$
\begin{aligned}
2 \psi^{(2)} & =\alpha a+\eta^{2} \psi_{x x}^{(2)}-6 \eta \psi^{(3)}-12 \eta^{2} \psi^{(4)}+O\left(\epsilon^{6}\right) \\
& =\alpha a+3 \eta^{2} \psi_{x x}^{(2)}-\eta p_{x}^{(0)}+O\left(\epsilon^{6}\right) .
\end{aligned}
$$

But from (85) and (41)

$$
p^{(0)}=\beta a-\eta p^{(1)}-4 \eta \psi_{x}^{(2)}+O\left(\epsilon^{5}\right)=\beta a-2 \eta \psi_{x}^{(2)}+O\left(\epsilon^{5}\right) .
$$

Hence, by combining (86) and (87), we obtain

$$
p^{(0)}=\beta a-\alpha \epsilon a_{x}+O\left(\epsilon^{5}\right)
$$

and

$$
\begin{aligned}
2 \psi^{(2)} & =\alpha a+3 \eta^{2} \psi_{x x}^{(2)}-\eta\left[\beta a-2 \eta \psi_{x}^{(2)}\right]_{x}+O\left(\epsilon^{6}\right) \\
& =\alpha a-\beta \epsilon a_{x}+\frac{5}{2} \epsilon^{2} \alpha a_{x x}+O\left(\epsilon^{6}\right)
\end{aligned}
$$


Substituting into (83), we finally obtain

$$
\epsilon^{2} a_{t}+\alpha \epsilon(\epsilon+2 a) a_{x}-\frac{1}{3} \beta \epsilon^{3} a_{x x}+\alpha \epsilon^{4} a_{x x x}=O\left(\epsilon^{8}\right) .
$$

It is seen that this equation differs from the Burgers' equation (72) by a term of third order derivative. However, it must be emphasized that Eq. (72) is not merely the approximation of Eq. (90) by omitting $\alpha \epsilon^{4} a_{x x x}$; since this term is of higher order than all the terms kept in (72) whereas it is of the same order as the nonlinear term kept in (90) (i.e., $\alpha \epsilon a a_{x}=O\left(\epsilon^{7}\right)$ ). This feature is already present in the classical nonlinear shallow water wave theory, where in the case of smaller amplitude (small but nonlinear effect still important) one has to bring in a higher order derivative. If the slope of the bed is small, e.g.,

$$
\alpha=\bar{\alpha} \epsilon \quad \bar{\alpha}=O(1),
$$

Eq. (90) can be approximated as

$$
\epsilon^{2} a_{\iota}+\bar{\alpha} \epsilon^{3} a_{x}=\frac{1}{3} \beta \epsilon^{3} a_{x x}+O\left(\epsilon^{8}\right),
$$

which is the heat equation for a moving medium.

The existence of permanent waves of the type

$$
a=a(\xi)=a(x-C t), \quad \xi=x-C t
$$

will certainly be of great interest. Changing $\partial / \partial t$ to $-C \partial / \partial \xi$ and $\partial / \partial x$ to $\partial / \partial \xi$ and integrating with respect to $\xi$ once, from $(90)$ we get

$$
(\alpha-C) \epsilon^{2} a+\alpha \epsilon a^{2}-\frac{1}{3} \beta \epsilon^{3} a_{\xi}+\alpha \epsilon^{4} a_{\xi \xi}=0 .
$$

We have again taken the integration constant to be zero so that $a_{\xi}=a_{\xi \xi}=0$ when $a=0$.

The appearance of a first derivative (negative "damping") in Eq. (94) now makes analytic solution infeasible in general, and we shall resort to the topological method on the phase plane (see e.g., Davis (1960)). Defining,

$$
a / \epsilon=\sigma, \quad \gamma=\xi / \epsilon \text { and } s=\sigma_{\gamma}
$$

we may rewrite Eq. (94) as

$$
\frac{d s}{d \sigma}=\frac{(\beta / 3 \alpha) s=\left[\sigma^{2}+(1-C / \alpha) \sigma\right]}{s}=\frac{(\beta / 3 \alpha) s-\sigma(\sigma-Z)}{s}
$$

with

$$
Z=(C / \alpha)-1
$$

We shall first assume $Z$ to be positive. Two singular points, $I$ and $I I$, appear in the phase plane $(\sigma, s)$ as shown in Fig. (5):

$$
I:(\sigma=0, s=0), \quad I I:(\sigma=Z, s=0)
$$

Following standard procedures, one finds point $I$ to be a saddle point. Three 
different types of singular behavior may be expected near $I I$, according as:

$$
\begin{array}{rll}
>0 & \text { Unstable node } \\
(\beta / 3 \alpha)^{2}-4 Z & =0 & \text { Unstable degenerate node } \\
& <0 & \text { Unstable spiral point. }
\end{array}
$$

Detailed study shows that the solution curve connecting these two singular points exists and is shown in Fig. (5a), (5b) and (5c). Clearly, $I(0,0)$ corresponds to the surface level far downstream and $I I(Z, 0)$ to the far upstream. The various profiles are drawn qualitatively in Fig. (6a) and (6b). While (99.a and 99.b) again give monoclinal waves, (99.c) now gives a profile with some undulations in the upstream which may be called the "polyclinical wave." The waviness becomes more and more pronounced towards the wave front which finally makes a decline to the downstream level.

The case of $Z=(C / \alpha)-1<0$ is similar. The singular points are now located at

$$
I:(\sigma=-|Z|, s=0) \text { and } I I:(\sigma=0, s=0),
$$

and hence they are shifted to the left by a distance $|(C / \alpha)-1|$ and the analysis can be performed just as before. Physically, the surface elevation changes from $\epsilon$ at far upstream to $\epsilon(1-|Z|)$ at far downstream. While the downstream point $(I)$ is again a saddle, the upstream point $(I I)$ has various singular behavior according to (99.a, b and c) if $Z$ is replaced by $|Z|$. In summary, we have

$$
\begin{aligned}
\text { monoclinal wave } & \geqq 0 \\
\text { if }(\beta / 3 \alpha)^{2}-4|Z| & \\
\text { polyclinal wave } & <0
\end{aligned}
$$

The "amplitude dispersion curve", $Z$ vs $C / \alpha$, is again a straight line (ef. Eq. (97)), same as the medium amplitude case.

Equation (101) also provides the condition whether corrugations may or may not occur on the surface of a highly viscous fluid flowing down an inclined plane. The criterion is that corrugations do not occur if

$$
\begin{gathered}
(\beta / 3 \alpha)^{2}-4|Z|=\frac{1}{9} \operatorname{Cot}^{2} \theta-4|Z| \geqq 0, \\
\theta \leqq \tan ^{-1}(1 / 6 \sqrt{|Z|})
\end{gathered}
$$

In general, the limiting angle can then be close to $\pi / 2$ because of the smallness of $Z$.

Of particular interest is the case of vertical wall where $\beta=0$ and $\alpha=1$. Equation (94) reduces to

$$
\sigma_{\gamma \gamma}+\sigma^{2}-(C-1) \sigma+A_{1}=0
$$



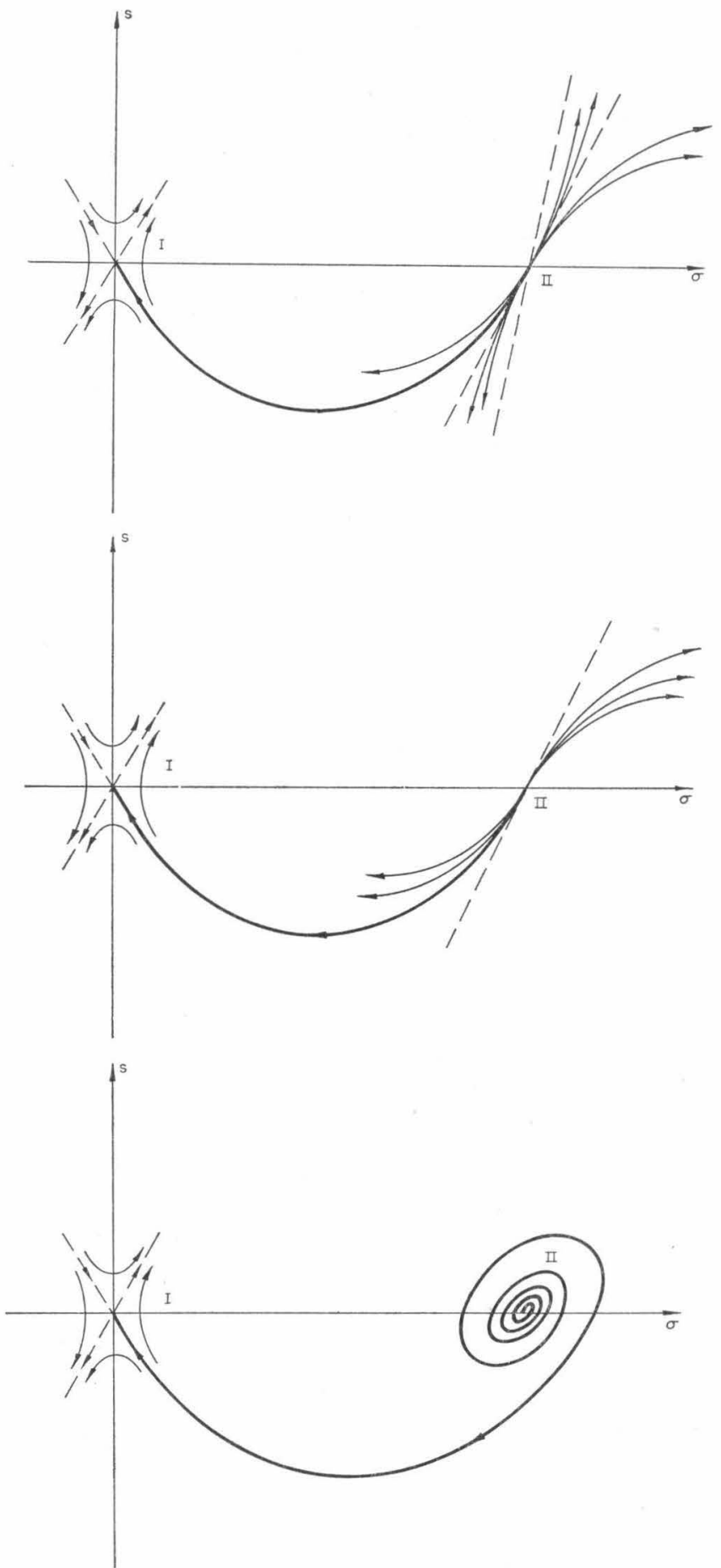

Fig. 5. Phase plane trajectories for small amplitude waves $\left(a=O\left(\epsilon^{3}\right)\right)$. a). Node to saddle. b). Degenerate node to saddle. c). Spiral point to saddle. 
or, upon integration,

$$
\begin{aligned}
\left(\sigma_{\gamma}\right)^{2} & =2\left[-\frac{1}{3} \sigma+\frac{1}{2}(C-1) \sigma^{2}-A_{1} \sigma+A_{2}\right] \\
& =\frac{2}{3}\left(\sigma_{1}-\sigma\right)\left(\sigma-\sigma_{2}\right)\left(\sigma-\sigma_{3}\right), \sigma_{1}>\sigma_{2}>\sigma_{3}
\end{aligned}
$$

in which we have kept the integration constants $A_{1}$ and $A_{2}$. With minor difference in the coefficients this is just the well known cnoidal wave equation. Its periodic solution, cnoidal wave, can be expressed as follows,

$$
\sigma=\sigma_{2}+\left(\sigma_{1}-\sigma_{2}\right) c n^{2}\left(\sqrt{\left(\sigma_{2}-\sigma_{3}\right) / 6} \gamma, k\right)
$$

with

$$
k=\sqrt{\left(\sigma_{1}-\sigma_{2}\right) /\left(\sigma_{1}-\sigma_{3}\right)}
$$

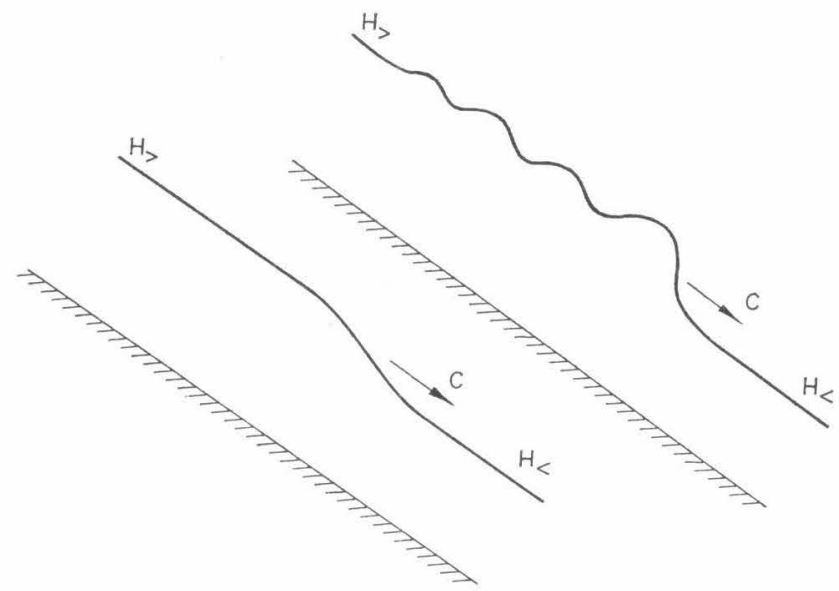

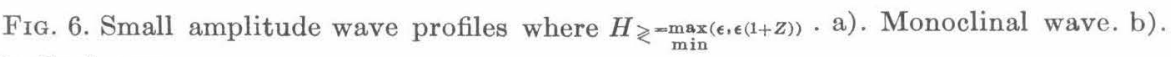
Polyelinal wave.

and the wavelength

$$
\lambda=\frac{2 \sqrt{6}}{\sqrt{\sigma_{1}-\sigma_{3}}} \int_{0}^{\frac{\pi}{2}} \frac{d \phi}{\sqrt{1-k^{2} \sin ^{2} \phi}} .
$$

Experiments regarding wave formation in a vertical sheet of liquid exist (see Binnie (1957) for a survey). Since they all indicate fairly large amplitudes, the present nonlinear theory may be of some relevance. However, due to the lack of measurements of wave amplitudes direct comparison of theory and experiments has not been made.

A cnoidal wave of infinite wavelength is the solitary wave which corresponds to the solution of Eq. (103) with $A_{1}=A_{2}=0$. The amplitude is given by the formula

$$
\sigma / \sigma_{0}=\operatorname{sech}^{2}\left(\sqrt{3 \sigma_{0} / 8} \gamma\right), \quad \sigma_{0}=\frac{3}{2}(C-1)
$$

where $\sigma_{0}$ is the maximum value of $\sigma$. Hence the highest elevation above the level 
at infinity is related to the wavespeed as,

$$
C=1+\frac{2}{3} a_{\max } / \epsilon .
$$

Finally, it may be remarked that in the case of $\beta \neq 0$ the effect of non-zero integration constants would be to lower or to heighten the surface levels at infinity and no significantly new feature is found.

Acknowledgment. The present research was supported by the U. S. Office of Naval Research under Contract Nonr-220(35) with California Institute of Technology. The author wishes to thank Professor T. Yao-Tsu Wu for helpful comments.

Appendix I. We present here an argument which shows that the choice of the time scale is the logical one. As is briefly explained near the end of $\$ 2$ and later demonstrated in $\$ \S 3,4$ and 5 , the approximate governing equation is obtained from the three boundary conditions $(15,16,17)$ on the free surface. An observation of these equations indicates that the time dependence appears explicitly in Eq. (15) only through the term $\tau \eta_{t}$. In order that non-trivial dynamical motion may be treated, one must choose the Strouhal number $\tau$ such that this term is equally important as the other first order terms. Expressing (15) in terms of expansions we have

$$
\tau \eta_{t}+\left\{\frac{1}{2} \alpha \epsilon^{2}\left[1-(a / \epsilon)^{2}\right]+2 \eta \psi^{(2)}+\cdots\right\} \eta_{x}+\eta^{2} \psi_{x}^{(2)}+\eta^{(3)} \psi_{x}^{3}+\cdots=0 .
$$

In view of (44), it is now clear that we should take $\tau=O\left(\epsilon^{2}\right)$. Without loss of generality Eq. (26) may be adopted from which the time scale (Eq. (25)) is derived.

Appendix II. Derivation of Recursive Relations. By differentiating the series (36), the following expressions are obtained:

$$
\left.\begin{array}{rl}
\psi_{y} & =\sum_{0} y^{n}(n+1) \psi^{(n+1)}, \quad \psi_{y y}=\sum_{0} y^{n}(n+1)(n+2) \psi^{(n+2)} \\
\psi_{y y y} & =\sum_{0} y^{n}(n+1)(n+2)(n+3) \psi^{(n+3)}
\end{array}\right\}
$$

with $\psi^{(0)}=\psi^{(1)}=0$. Written in terms of $\psi$, the momentum equations are

$$
\epsilon^{3} \psi_{y t}+\epsilon\left[\left(U+\psi_{y}\right) \psi_{x y}-\psi_{x}\left(U_{y}+\psi_{y y}\right)\right]=-p_{x}+\psi_{y y y}+\psi_{y x x}
$$

and

$$
\epsilon^{3} \psi_{x t}+\epsilon\left[\left(U+\psi_{y}\right) \psi_{x x}-\psi_{x} \psi_{x y}\right]=p_{y}+\psi_{x x x}+\psi_{x y y} .
$$

Substituting the series (36) and (37) in (b) and making use of (a), we have

$$
\begin{aligned}
& \epsilon^{3} \sum y^{n}(n+1) \psi_{t}^{(n+1)}+\epsilon\left(\alpha \epsilon y-\frac{1}{2} \alpha y^{2}\right) \sum y^{n}(n+1) \psi_{x}^{(n+1)} \\
& +\epsilon\left[\sum y^{n}(n+1) \psi^{(n+1)}\right]\left[\sum y^{n}(n+1) \psi_{x}^{(n+1)}\right]-\epsilon(\alpha \epsilon-\alpha y) \sum y^{n} \psi_{x}^{(n)} \\
& -\epsilon\left[\sum y^{n} \psi_{x}^{(n)}\right]\left[\sum y^{n}(n+1)(n+2) \psi^{(n+2)}\right] \\
& =-\sum y^{n} p_{x}^{(n)}+\sum y^{n}(n+1)\left[\psi_{x x}^{(n+1)}+(n+2)(n+3) \psi^{(n+3)}\right] .
\end{aligned}
$$


Since this must hold for arbitrary $O \leqq y \leqq \eta$, the coefficient of each power of $y$ vanishes individually. Hence, from

$$
y^{0}: p_{x}^{(0)}=6 \psi^{3)}
$$

and

$$
y^{1}: 2 \epsilon^{3} \psi_{t}^{(2)}+p_{x}^{(1)}=2 \psi_{x x}^{(2)}+24 \psi^{(4)}, \text { etc. }
$$

Eq. (c) can likewise be written in series as follows,

$$
\begin{gathered}
\epsilon^{3} \sum y^{n} \psi_{x t}^{(n)}+\epsilon\left(\alpha \epsilon y-\frac{1}{2} \alpha y^{2}\right) \sum y^{n} \psi_{x x}^{(n)}+\epsilon\left[\sum y^{n}(n+1) \psi^{(n+1)}\right]\left[\sum y^{n} \psi_{x x}^{(n)}\right] \\
-\epsilon\left[\sum y^{n} \psi_{x}^{(n)}\right]\left[\sum y^{n}(n+1) \psi_{x}^{(n+1)}\right] \\
=\sum y^{n}(n+1) p^{(n+1)}+\sum y^{n}\left[\psi_{x x x}^{(n)}+(n+1)(n+2) \psi_{x}^{(n+2)}\right]
\end{gathered}
$$

from which,

$$
y^{0}: 0=p^{(1)}+2 \psi_{x}^{(2)}
$$

and

$$
y^{1}: 0=2 p^{(2)}+6 \psi_{x}^{(3)}, \text { etc. }
$$

Equations (40)-(44) follow immediately from (e), (f), (h) and (i).

\section{REFERENCES}

Benjamin, T. B., (1957), Wave formation in laminar flow down an inclined plane. J. Fluid Mech. 2, 554-574.

Benjamin, T. B., (1962), The solitary wave on a stream with an arbitrary distribution of vorticity. J. Fluid Mech. 12, 97-116.

Binnie, A. M., (1957), Experiments on the onset of wave formation on a film of water flowing down a vertical plane. J. Fluid Mech. 2, 551-553.

Burns, J. C., (1953), Long waves in running water, (Appendix by M. J. Lighthill). Proc. Camb. Philo. Soc. 49, 695-706.

Cole, J. D., (1951), On a quasi-linear parabolic equation occurring in aerodynamics. Q. Appl. Math. 9, 225-236.

Courant, R. and D. Hilbert, (1962), Methods of mathematical physics, II, Interseience, New York.

DAvis, H. T., (1960), Introduction to nonlinear differential and integral equations. Dover.

Dressler, R. F., (1949), Mathematical solution of the problem of roll-waves in inclined open-channels. Comm. Pure Appl. Math. 2, 149-194.

Hopf, E., (1950), The partial differential equation $u_{t}+u u_{x}=\mu u_{x x}$. Comm. Pur Appl. Math. 3, 201-230.

Hunt, J. N., (1955), Gravity waves in flowing water. Proc. Roy. Soc. London, 231A, 496504.

Jeffrex, A. And Taniuti, T., (1964), Non-linear wave propagation, Academic Press, New York.

Keller, J. B., (1948), The solitary and periodic waves in shallow water. Comm. Pure Appl. Math. 1, 323-339.

LAx, P. D., (1957, Hyperbolic systems of conservation laws, II. Comm. Pure Appl. Math. $10,537-566$.

Lighthill, M. J., (1956), Viscosity effects in sound waves of finite amplitude in Survey in Mechanics ed. G. K. Batchelor and R. M. Davies, Cambridge.

Lighthill, M. J. and Whitham, G. B., (1955), On kinematic waves, I. Flood movement in 
long waves, II. Theory of traffic flow on long crowded roads. Proc. Roy Soc. London, 229A, 281-345.

Lin, C. C. And Clark, Jr., Alfred, (1959), On the theory of shallow water waves. Tsing Hua J. of Chinese Studies, 55-61.

Longuet-Higgins, (1958), Review on Water waves by J. J. Stoker, J. Fluid Mech. 4, 435439.

Stoker, J. J., (1957), Water waves, Interscience, New York.

Ursell, F., (1953), The long wave paradox in the theory of gravity waves. Proc. Camb. Philo. Soc. 49, 685-694.

Wehausen, J. V. and Laitone, E. V., (1960), Surface waves in Handbuch der Physik. Springer-Verlag. 311-758.

YIH, C. S., (1954), Stability of parallel laminar flow with a free surface. Proc. 2nd U. S. Nat'l. Congr. Appl. Mech., 623-628.

YIH, C. S., (1963), Stability of liquid flow down an inclined plane. Phys. Fluids 6, 321-334.

Department of Civil Engineering

Massachusetts Institute of Technology

Cambridge, Massachusetts

(Received July 9, 1965) 
DISTRIBUTION LIST FOR UNCLASSIFIED TECHNICAL REPORTS ISSUED UNDER

CONTRACT Nonr-220(35)

(Single copy unless otherwise specified)

Chief of Naval Research

Department of the Navy

Washington 25, D. C.

Attn: Codes 438 (3)

461

463

466

Commanding Officer

Office of Naval Research

Branch Office

495 Summer Street

Boston 10, Massachusetts

Commanding Officer

Office of Naval Research

Branch Office

207 West 24th Street

New York 11, New York

Commanding Officer

Office of Naval Research

Branch Office

1030 East Green Street

Pasadena, California

Commanding Officer

Office of Naval Research

Branch Office

1076 Mission Street

San Francisco, California 94103

Commanding Officer

Office of Naval Research

Branch Office

Box 39, Navy No. 100

Fleet Post Office

New York, New York (25)

Director

Naval Research Laboratory

Washington 25, D. C.

Attn: Code 2027 (6)

Chief, Bureau of Naval Weapons

Department of the Navy

Wa shington 25, D. C.

Attn: Codes RUAW-r

RRRE

RAAD

RAAD-222

DIS -42
Commander

U. S. Naval Ordnance Test Station

China Lake, California

Attn: Code 753

Chief, Bureau of Ships

Department of the Navy

Wa shington 25, D. C.

Attn: Codes 310

312

335

420

421

440

442

449

Chief, Bureau of Yards and Docks Department of the Navy

Wa shington 25, D. C.

Attn: Code D-400

Commanding Officer and Director David Taylor Model Basin

Wa shington 7, D. C.

Attn: Codes 108

142

500

513

520

525

526

$526 \mathrm{~A}$

530

533

580

585

589

591

$591 \mathrm{~A}$

700

Commander

U. S. Naval Ordnance Test Station

Pasadena Annex

3202 E. Foothill Blvd.

Pasadena 8, California

Attn: Code P-508

Commander

Planning Department

Portsmouth Naval Shipyard

Portsmouth, New Hampshire 
Commander

Planning Department

Boston Naval Shipyard

Boston 29, Massachusetts

Commander

Planning Department

Pearl Harbor Naval Shipyard

Navy No. 128, Fleet Post Office

San Francisco, California

Commander

Planning Department

San Francisco Naval Shipyard

San Francisco 24, California

Commander

Planning Department

Mare Island Naval Shipyard

Vallejo, California

Commander

Planning Department

New York Naval Shipyard

Brooklyn 1, New York

Commander

Planning Department

Puget Sound Naval Shipyard

Bremerton, Washington

Commander

Planning Department

Philadelphia Naval Shipyard

U. S. Naval Base

Philadelphia 12, Pennsylvania

Commander

Planning Department

Norfolk Naval Shipyard

Portsmouth, Virginia

Commander

Planning Department

Charleston Naval Shipyard

U. S. Naval Base

Charleston, South Carolina

Commander

Planning Department

Long Beach Naval Shipyard

Long Beach 2, California

Commander

Planning Department

U. S. Naval Weapons Laboratory

Dahlgren, Virginia

Commander

U. S. Naval Ordnance Laboratory

White Oak, Maryland
Dr. A. V. Hershey

Computation and Exterior

Ballistics Laboratory

U. S. Naval Weapons Laboratory

Dahlgren, Virginia

Superintendent

U. S. Naval Academy

Annapolis, Maryland

Attn: Library

Superintendent

U. S. Naval Postgraduate School

Monterey, California

Commandant

U. S. Coast Guard

1300 E Street, N. W.

Washington, D. C.

Secretary Ship Structure Committee

U. S. Coast Guard Headquarters

1300 E Street, N. W.

Wa shington, D. C.

Commander

Military Sea Transportation Service

Department of the Navy

Wa shing ton 25, D. C.

U. S. Maritime Administration

GAO Building

441 G Street, N. W.

Wa shington, D. C.

Attn: Division of Ship Design

Division of Research

Superintendent

U. S. Merchant Marine Academy

Kings Point, Long Island, New York

Attn: Capt. L. S. McCready

(Department of Engineering)

Commanding Officer and Director

U. S. Navy Mine Defense Laboratory

Panama City, Florida

Commanding Officer

NROTC and Naval Administrative

Massachusetts Institute of Technology

Cambridge 39, Massachusetts

U. S. Army Transportation Research

and Development Command

Fort Eustis, Virginia

Attn: Marine Transport Division

Mr. J. B. Parkinson

National Aeronautics and

Space Administration

1512 H Street, N. W.

Wa shington $25, \mathrm{D}$. C. 


\author{
Dir ector \\ Langley Research Center \\ Langley Station \\ Hampton, Virginia \\ Attn: Mr. I. E. Garrick \\ Mr. D. J. Marten
}

Director Engineering Science Division National Science Foundation

1951 Constitution Avenue, N. W.

Washington 25, D. C.

Dir ector

National Bureau of Standards

Wa shington 25 , D. C.

Attn: Fluid Mechanics Division

(Dr. G. B. Schubauer)

Dr. G. H. Keulegan

Dr. J. M. Franklin

Defense Documentation Center

Cameron Station

Alexandria, Virginia (20)

Scientific and Technology Division

Library of Congress

Washington, D. C. 20540

California Institute of Technology

Pasadena 4, California

Attn: Professor M. S. Plesset

Professor T. Y. Wu

Professor A. J. Acosta

University of California

Department of Engineering

Los Angeles 24, California

Attn: Dr. A. Powell

Director

Scripps Institute of Oceanography

University of California

La Jolla, California

Professor M. L. Albertson

Department of Civil Engineering

Colorado $\mathrm{A}$ and $\mathrm{M}$ College

Fort Collins, Colorado

Professor J. E. Cernak

Department of Civil Engineering

Colorado State University

Fort Collins, Colorado

Professor W. R. Sears

Graduate School of

Aeronautical Engineering

Cornell University

Ithaca, New York

State University of Iowa

Iowa Institute of Hydraulic Research

Iowa City, Iowa

Attn: Dr. H. Rouse

Dr. L. Landweber
Massachusetts Institute of Technology

Cambridge 39, Massachusetts

Attn: Department of Naval Architecture and Marine Engineering Professor A. T. Ippen

Harvard University

Cambridge 38, Massachusetts

Attn: Professor G. Birkhoff (Department of Mathematics)

Professor G. F. Carrier

(Department of Mathematics)

University of Michigan

Ann Arbor, Michigan

Attn: Professor R. B. Couch (Department of Naval Architecture)

Professor W. W. Willmarth (Department of Aeronautical Engineering)

Dr. L. G. Straub, Director

St. Anthony Falls Hydraulic Laboratory

University of Minnesota

Minneapolis 14, Minnesota

Attn: Mr. J. N. Wetzel

Professor B. Silberman

Professor J. J. Foody

Engineering Department

New York State University

Maritime College

Fort Schylyer, New York

New York Univer sity

Institute of Mathematical Sciences

25 Waverly Place

New York 3, New York

Attn: Professor J. Keller Professor J. J. Stoker

The Johns Hopkins University

Department of Mechanical Engineering

Baltimore 18, Maryland

Attn: Professor S. Corrsin

Professor O. M. Phillips (2)

Massachusetts Institute of Technology

Department of Naval Architecture and

Marine Engineering

Cambridge 39, Massachusetts

Attn: Professor M. A. Abkowitz

Dr. G. F. Wislicenus

Ordnance Research Laboratory

Pennsylvania State University

University Park, Pennsylvania

Attn: Dr. M. Sevik

Professor R. C. DiPrima

Department of Mathematics

Rensselaer Polytechnic Institute

Troy, New York 
Director

Woods Hole Oceanographic Institute

Woods Hole, Massachusetts

Stevens Institute of Technology

Davidson Laboratory

Castle Point Station

Hoboken, New Jersey

Attn: Mr. D. Savitsky

Mr. J. P. Breslin

Mr. C. J. Henry

Mr. S. Tsakonas

Webb Institute of Naval Architecture

Crescent Beach Road

Glen Cove, New York

Attn: Professor E. V. Lewis

Technical Library

Executive Director

Air Force Office of Scientific Research

Washington 25, D. C.

Attn: Mechanics Branch

Commander

Wright Air Development Division

Aircraft Laboratory

Wright-Patterson Air Force Base, Ohio

Attn: Mr. W. Mykytow,

Dynamics Branch

Cornell Aeronautical Laboratory

4455 Genesee Street

Buffalo, New York

Attn: Mr. R. White

Massachusetts Institute of Technology

Fluid Dynamics Research Laboratory

Cambridge 39, Massachusetts

Attn: Professor H. Ashley

Professor M. Landahl

Professor J. Dugundji

Hamburgische Schiffbau-Versuchsanstalt Bramfelder Strasse 164

Hamburg 33, Germany

Attn: Dr. H. Schwanecke

Dr. H. W. Lerbs

Institut fur Schiffbau der

Universitat Hamburg

Berliner Tor 21

Hamburg 1, Germany

Attn: Professor G. P. Weinblum

Max-Planck Institut fur Stromungsfors chung Bottingerstrasse 6/8

Gottingen, Germany

Attn: Dr. H. Reichardt
Hydro-og Aerodynamisk Laboratorium Lyngby, Denmark

Attn: Professor Carl Prohaska

Skipsmodelltanken

Trondheim, Norway

Attn: Professor J. K. Lunde

Versuchsanstalt fur Wasserbau and Schiffbau

Schleuseninsel im Tiergarten

Berlin, Germany

Attn: Dr. S. Schuster, Director Dr. Grosse

Technische Hogeschool

Institut voor Toegepaste Wiskunde

Julianalaan 132

Delft, Netherlands

Attn: Professor R. Timman

Bureau D'Analyse et de Recherche Appliquees

47 Avenue Victor Bresson

Issy-Les-Moulineaux

Seine, France

Attn: Professor Siestrunck

Netherlands Ship Model Basin

Wageningen, The Netherlands

Attn: Dr. Ir. J. D. van Manen

National Physical Laboratory Teddington, Middlesex, England

Attn: Mr. A. Silverleaf, Superintendent Ship Division Head, Aerodynamics Division

Head, Aerodynamics Department Royal Aircraft Establishment Farnborough, Hants, England Attn: Mr. M. O. W. Wolfe

Dr. S. F. Hoerner

148 Busteed Drive

Midland Park, New Jersey

Boeing Airplane Company

Seattle Division

Seattle, Washington

Attn: Mr. M. J. Turner

Electric Boat Division

General Dynamics Corporation

Groton, Connecticut

Attn: Mr. Robert McCandliss

General Applied Sciences Labs., Inc. Merrick and Stewart Avenues

Westbury, Long Island, New York

Gibbs and Cox, Inc.

Technical Information Control Section

21 West Street

New York, N. Y. 10006 
Lockheed Aircraft Corporation

Missiles and Space Division

Palo Alto, California

Attn: R. W. Kermeen

Grumman Aircraft Engineering Corp.

Bethpage, Long Island, New York

Attn: Mr. E. Baird

Mr. E. Bower

Mr. W. P. Carl

Midwest Research Institute

425 Volker Blvd.

Kansas City 10 Missouri

Attn: Mr. Zeydel

Director, Department of Mechanical

Sciences

Southwest Research Institute

8500 Culebra Road

San Antonio 6, Texas

Attn: Dr. H. N. Abramson

Mr. G. Ransleben

Editor, Applied Mechanics

Review

Convair

A Division of General Dynamics

San Diego, California

Attn: Mr. R. H. Oversmith

Mr. H. T. Brooke

Hughes Tool Company

Aircraft Division

Culver City, California

Attn: Mr. M. S. Harned

Hydronautics, Incorporated

Pindell School Road

Howard County

Laurel, Maryland

Attn: Mr. Phillip Eisenberg

Rand Development Corporation

13600 Deise Avenue

Cleveland 10, Ohio

Attn: Dr. A. S. Iberall

U. S. Rubber Company

Research and Development Department

Wayne, New Jersey

Attn: Mr. L. M. White

Technical Research Group, Inc.

Route 110

Melville, New York, 11749

Attn: Mr. Jack Kotik

Mr. C. Wigley

Flat 102

6-9 Charterhouse Square

London, E. C. 1, England
AVCO Corporation

Lycoming Division

1701 K Street, N. W.

Apt. No. 904

Washington, D. C

Attn: Mr. T. A. Duncan

Mr. J. G. Baker

Baker Manufacturing Company

Evansville, Wisconsin

Curtiss-Wright Corporation Research

Division

Turbomachinery Division

Quehanna, Pennsylvania

Attn: Mr. George H. Pedersen

Dr. Blaine R. Parkin

General Dynamics/Convair

Plant 71 P. O. 1128

San Diego, California

The Boeing Company

Aero-Space Division

Seattle 24, Washington

Attn: Mr. R. E. Bateman

Internal Mail Station 46-74

Lockheed Aircraft Corporation

California Division

Hydrodynamics Research

Burbank, California

Attn: Mr. Bill East

National Research Council

Montreal Road

Ottawa 2, Canada

Attn: Mr. E. S. Turner

The Rand Corporation

1700 Main Street

Santa Monica, California

Attn: Technical Library

Stanford University

Department of Civil Engineering

Stanford, California

Attn: Dr. Byrne Perry

Dr. E. Y. Hsu

Dr. Hirsh Cohen

IBM Research Center

P. O. Box 218

Yorktown Heights, New York

Mr. David Wellinger

Hydrofoil Projects

Radio Corporation of America

Burlington, Massachusetts

Food Machinery Corporation

P. O. Box 367

San Jose, California

Attn: Mr. G. Tedrew 
Dr. T. R. Goodman

Oceanics, Inc.

Technical Industrial Park

Plainview, Long Island, New York
The Transportation Technical Research Institute Investigation Office Ship Research Institute 700 Shinkawa, Mitaka

Tokyo, Japan

\section{Commanding Officer}

Office of Naval Research

Branch Office

219 S. Dearborn Street

Chicago 1, Illinois 60604

University of Colorado

Aerospance Engineering Sciences

Boulder, Colorado

Attn: Professor M. S. Uberoi

The Pennsylvania State University

Department of Aeronautical

Engineering

Ordnance Research Laboratory

P. O. Box 30

State College, Pennsylvania

Attn: Professor J. William Holl

Institut fur Schiffbau der

Universitat Hamburg

Lammersieth 90

2 Hamburg 33, Germany

Attn: Dr. O. Grim

Technische Hogeschool

Laboratorium voor Scheepsbounkunde

Mekelweg 2, Delft, The Netherlands

Attn: Professor Ir. J. Gerritsma

Dr. I. S. Pearsall

National Engineering Laboratory

Glasgow, Scotland

Prof. Jacques Dodu

Maitre de Conferences a la Faculte

des Sciences

Laboratoires de Mecanique des Fluides

44-46, Avenue Felix-Viallet

Grenoble (Isere), France

Dr. Karlo K. Mustonen

Head - Documents Division

Utah State University

Logan, Utah 84321

Dr. James W. Daily

Department of Engineering Mechanics

College of Engineering

The University of Michigan

Ann Arbor, Michigan, 48104 